\title{
Colour-dependent accurate modelling of dynamical parallaxes and masses of visual binaries
}

\section{Application to the VB+SB2 systems with definitive orbits}

\author{
Manuel Andrade ${ }^{1,2}$ \\ 1 Departamento de Matemática Aplicada, Instituto de Matemáticas, Escola Politécnica Superior de Engenharia, Universidade de \\ Santiago de Compostela (USC), Campus Terra, 27002 Lugo, Galiza, Spain \\ e-mail: manuel. andrade@usc.gal \\ 2 R. M. Aller Astronomical Observatory, USC, Campus Vida, 15782 Santiago de Compostela, Galiza, Spain
}

Received 27 June 2019 / Accepted 31 July 2019

\begin{abstract}
Context. Two fundamental characteristics of binary systems are the masses of their components and their distance to the Earth. In this way, the dynamical parallax is an accurate and very helpful tool. Nevertheless, there has been some concern with regard to the use of a unique linear mass-luminosity relation (MLR) for the entire main sequence (MS).

Aims. This article describes the accurate computation of both dynamical parallaxes and individual masses of visual binaries. The main aim is to formulate a model which would be suitable for binary systems attending to the exact locations of the components on the MS in the HR diagram.

Methods. An analytical model was developed which allows calculation of dynamical parallaxes and individual masses using a non-linear MLR valid for the entire MS. This up-to-date MLR is given by a polynomial of degree 20 . In contrast to previous approaches, this model can be applied even in the case of components with unequal masses, that is, with an arbitrarily large difference of magnitudes between them. On the other hand, considering the fundamental equation of the theory that forms the basis of the model, we propose to estimate uncertainties in parallax and masses using Monte Carlo simulations.

Results. The model was validated by means of numerical tests using a synthetic sample comprising $10^{3}$ systems. The results are much more accurate than those for previous models reported in the literature for deriving dynamical parallaxes and masses. Furthermore, we present dynamical parallaxes and individual masses for the 19 double-lined spectro-interferometric systems with definitive visual orbits and compare the former with the orbital parallaxes as well as with those measured by HIPPARCos and Gaia. It is worth mentioning that the latter can only be a reliable source when the orbital motion is taken into account. Thus, at present, many Gaia DR2 parallaxes of binaries are biased.

Conclusions. Our model, composed of an exact analytical theory, along with a non-linear MLR, guarantees high accuracy even in cases where the components are of unequal mass.
\end{abstract}

Key words. methods: analytical - methods: statistical - astrometry - parallaxes - binaries: visual - stars: fundamental parameters

\section{Introduction}

The pioneering works of Russell \& Moore (1940) and Baize \& Romani (1946) laid the foundation for the use of the massluminosity relation (MLR) along with Kepler's third law for calculating the dynamical parallax and the component masses of a main sequence (MS) visual binary. Although both approaches are equivalent, the Baize-Romani method has been more widely used over the years because of its simplicity and ease of use with different types of mass-luminosity arrays,

$\log \mathcal{M}=-\frac{2}{5} \kappa\left(M_{\mathrm{bol}}-M_{0}\right)$,

with $\mathcal{M}$ being the mass of the binary system in solar masses, $M_{\text {bol }}$ its absolute bolometric magnitude, and $\kappa$ and $M_{0}$ two constants; the second is indeed the bolometric magnitude of the Sun. In addition, the absolute bolometric magnitude is related to the parallax by means of the distance modulus,

$m_{\text {bol }}-M_{\text {bol }}=-5(1+\log \varpi)$,

where $m_{\text {bol }}$ is the apparent bolometric magnitude of the star and $\varpi$ is the parallax in arcseconds. This procedure was subsequently adapted to systems containing an unresolved subsystem by Heintz (1962).

On the other hand, the only serious criticism of this method has probably been that of its applicability to binary systems with components over the entire MS. In fact, although Couteau (1978) demonstrated that the method is not very sensitive to small deviations from the MLR, mainly for G-type stars, we cannot completely rule out the existence of significant residuals for other spectral types.

This drawback can be, however, fully overcome considering a non-linear MLR valid for the entire MS. This was partially accomplished by Angelov (1993), who suggested the use of an MLR given by a power series. Unfortunately, his assumption of equal-mass components in the expansion in series near to the origin of an intermediate function yields a (linear) first-order approximation that neglects all the contributions owing to the difference between the masses of the components. Nevertheless, this is an indispensable condition in his development, because he is using non-convergent series.

In the present work, we assume a non-linear MLR obtained for the entire MS, and develop a new theory which is valid for 
unequal-mass components and that additionally takes interstellar extinction into account. This is an exact theory, without expansions in series, that allows to obtain rigorous equations for both the dynamical parallax and the individual dynamical masses. Additionally, it is used to provide accurate parallaxes and masses of a representative sample of 19 double-lined spectro-interferometric binaries $(\mathrm{VB}+\mathrm{SB} 2)$.

This paper is organised as follows. Our analytical theory is derived in Sect. 2. An up-to-date fit of the MLR valid for the entire MS is given in Sect. 3. In the fourth section, a case study is presented to illustrate the numerical resolution for our model. Furthermore, a synthetic sample of $10^{3}$ systems is used to validate the model as well as to highlight its noticeably higher accuracy in comparison with previous ones. The application of the model to the $19 \mathrm{VB}+\mathrm{SB} 2$ binary systems with definitive orbits in the Sixth Catalog of Orbits of Visual Binary Stars (Hartkopf et al. 2001), hereafter ORB6, is discussed in Sect. 5, where a Monte Carlo simulation is implemented to obtain reliable uncertainties both in parallax and in individual masses. Finally, the conclusions are outlined in Sect. 6.

\section{Analytical theory}

\subsection{Basic equations}

We use Kepler's third law,

$\mathcal{M}_{1}+\mathcal{M}_{2}=\left(\frac{a}{\varpi}\right)^{3} \frac{1}{P^{2}}$

where $\mathcal{M}_{i}$, with $i=1,2$, is the mass of each component of the binary system (in solar masses), $a$ the orbital semimajor axis (in arcseconds), and $P$ the orbital period (in years). Now, we define a non-linear mass-luminosity relation

$\log \mathcal{M}=\sum_{k=0}^{n} c_{k} M_{\mathrm{bol}}^{k}$,

where $c_{k}$ are coefficients obtained from a fit $\left(\log \mathcal{M}, M_{\mathrm{bol}}\right)$ along the MS (see Sect. 3.1.2).

Thus, Expressions (3) and (4), together with the distance modulus expression given in Eq. (2), are the three basic equations of the theory. We rewrite the latter for each component $i$ as

$M_{\mathrm{bol}, i}=m_{\mathrm{V}, i}+\mathrm{BC}_{i}-A_{\mathrm{V}}+5+5 \log \varpi$,

where we introduce the bolometric correction (BC) to obtain the apparent bolometric magnitude from the apparent visual magnitude $\left(m_{\mathrm{V}}\right)$. Moreover, we have also taken the interstellar extinction $\left(A_{\mathrm{V}}\right)$ into account.

Therefore, a new mass-luminosity relation for each component $i$ can be obtained substituting (5) in (4),

$\log \mathcal{M}_{i}(y)=c_{0}+S_{i}(y)$,

where

$S_{i}(y)=\sum_{k=1}^{n} c_{k} \sum_{j=0}^{k}\left(\begin{array}{l}k \\ j\end{array}\right) x_{i}^{k-j} y^{j}$,

with $\left\{\begin{array}{l}x_{i}=m_{\mathrm{V}, i}+\mathrm{BC}_{i}-A_{\mathrm{V}}+5, \\ y=5 \log \varpi .\end{array}\right.$

Combining this result with Kepler's third law given in (3), we obtain

$\mathcal{M}_{i}(y) D_{i}(y)=h 10^{-0.6 y}$, where

$\left\{\begin{array}{l}h=a^{3} P^{-2} \\ D_{i}(y)=1+10^{-\alpha \Delta S(y)}\end{array}\right.$

with

$\Delta S(y) \equiv S_{2}(y)-S_{1}(y)=\log \frac{\mathcal{M}_{2}}{\mathcal{M}_{1}}$,

and $\alpha=-1^{i}$.

Taking logarithms in (8), we derive the fundamental equation

$\log \mathcal{M}_{i}(y)+\log D_{i}(y)=\log h-0.6 y$.

The main difference with the Baize-Romani method is that in this case the auxiliary function, $\log D_{i}(y)$, is a parallaxdependent function. In contrast with the Angelov's approach, we propose to solve Eq. (10) exactly, without using the near-origin expansion in series

$$
\begin{aligned}
\log D_{i}= & \log 2-\frac{\alpha}{2} \Delta S \\
& +\frac{\log 10}{8} \Delta S^{2}+\frac{\log ^{5} 10}{2880} \Delta S^{6}+O\left(\Delta S^{8}\right) .
\end{aligned}
$$

In such an approach, it is assumed that the masses of both components are equal (see Eq. (9)). This is necessary in order to remove all the non-convergent terms, that is, the terms of order higher than one $\left(O\left(\Delta S^{p}\right)=0\right.$ with $\left.p \geq 2\right)$. On the contrary, our theory can be applied without any assumption, thus avoiding any eventual overestimation of the parallax (see Sect. 4.2.2).

\subsection{The fundamental equation of the dynamical parallax}

We rewrite the Eq. (10) as

$\mathcal{U}(y)+\mathcal{V}(y)=0$

where

$\left\{\begin{array}{l}\mathcal{U}(y)=\log \left(1+10^{S_{2}(y)-S_{1}(y)}\right), \\ \mathcal{V}(y)=c_{0}+S_{1}(y)+0.6 y-\log h .\end{array}\right.$

This is a transcendental equation, the roots of which can be found by means of root-finding algorithms. In this case, we apply the Brent-Dekker method (Dekker 1969; Brent 1971), a robust and fast algorithm that works very well when one is dealing with a one-dimensional problem, the roots of which can be bracketed. In this case, the space of all feasible solutions of this equation, $\Omega=\{y \in \mathbb{R} \mid \mathcal{U}(y)+\mathcal{V}(y)=0\}$, is bounded. It is straightforward to verify this by taking the Expression (7) into account, along with the empirical fact that $\varpi<1$ arcsecond; thus the allowed solutions of Eq. (12) must be $y \in \mathbb{R}^{-}$. In addition, if we generously assume that we are searching dynamical parallaxes larger than $1 \mu$ as, we can consider $\Omega \subset(-30,0)$. Thus, the dynamical parallax is easily derived from the solution of (12) by means of $\varpi=10^{y / 5}$.

We note that the exact resolution of the fundamental equation using the Brent-Dekker method allows numerical accuracies in the dynamical parallax better than $1 \mu$ as to be achieved quickly. As a matter of fact, given the strong non-linearity of the fundamental Eq. (12), the demands on accuracy can be much more difficult to meet with other numerical methods. 
Table 1. Fits of the linear (MS) MLR customarily used to compute dynamical parallaxes by means of the classical Baize-Romani method.

\begin{tabular}{cccc}
\hline \hline Fit & $M_{0}$ & $\kappa$ & Spectral range \\
\hline I & 4.77 & $3.58 \pm 0.03$ & B0-G1 \\
II & $4.89 \pm 0.14$ & $3.88 \pm 0.08$ & O7.5-M4 \\
III & $4.74 \pm 0.05$ & $4.23 \pm 0.06$ & B3-M1 \\
IV & $4.740 \pm 0.083$ & $3.664 \pm 0.046$ & O7-M9.5 \\
\hline
\end{tabular}

References. (I) Baize \& Romani (1946); (II) McCluskey \& Kondo (1972); (III) Docobo \& Andrade (2013); (IV) this paper.

\subsection{Formulae for the dynamical masses}

Therefore, the solution of the Eq. (12), along with the Expressions (6) and (7), provide direct formulae for the mass of each component,

$\mathcal{M}_{1}=10^{c_{0}+S_{1}(y)}$

$\mathcal{M}_{2}=10^{c_{0}+S_{2}(y)}$.

Finally, we would like to point out that, as Heintz (1971) already indicated, we should only use the term dynamical with those parallaxes which are derived using both an orbit and an assumed MLR. Hence, parallaxes based on an assumed mass (e.g. using spectral types), apart from being inferior, are not genuine dynamical parallaxes.

\section{Photometric issues}

New linear and non-linear MLRs that are valid for the entire MS are provided in this section. The latter will be used together with the analytical theory described in Sect. 2 to compute accurate dynamical parallaxes and masses (see Sect. 4). In addition, the interstellar extinction law used in these computations is also introduced.

\subsection{The mass-luminosity relation}

\subsubsection{A new linear mass-luminosity relation}

The MLR plays a fundamental role in binary star astronomy since it allows the computation of dynamical parallaxes and individual masses for MS binary systems. Therefore, different sets of parameters (see Table 1) fitting a logarithmic linear MLR, such as that given in Eq. (1), have been considered in the past (see Baize \& Romani 1946; McCluskey \& Kondo 1972; Docobo \& Andrade 2013), each of them derived from different data sources.

With the aim of providing a reliable linear MLR, a new fit of the $\left(\log \mathcal{M}, M_{\text {bol }}\right)$ data has been carried out here, taking 88 masses and absolute bolometric magnitudes given by Pecaut $\&$ Mamajek (2013) into account ${ }^{1}$. A straight line with a coefficient of determination of $R^{2}=0.9910$ was obtained. A major advantage of this is that it is valid for a very wide spectral range of the MS (O7 V-M9.5 V). We have considered the need to use a bolometric magnitude of the Sun consistent with the zeropoint of the empirical bolometric corrections for stars (see Torres 2010). Parameters of this fitting, together with those mentioned above, are shown in Table 1. The linear fit is shown in Fig. 1.

1 Seehttp://www.pas.rochester.edu/ emamajek/EEM_dwarf_ UBVIJHK_colors_Teff.txt, updated as of 22 March 2019.

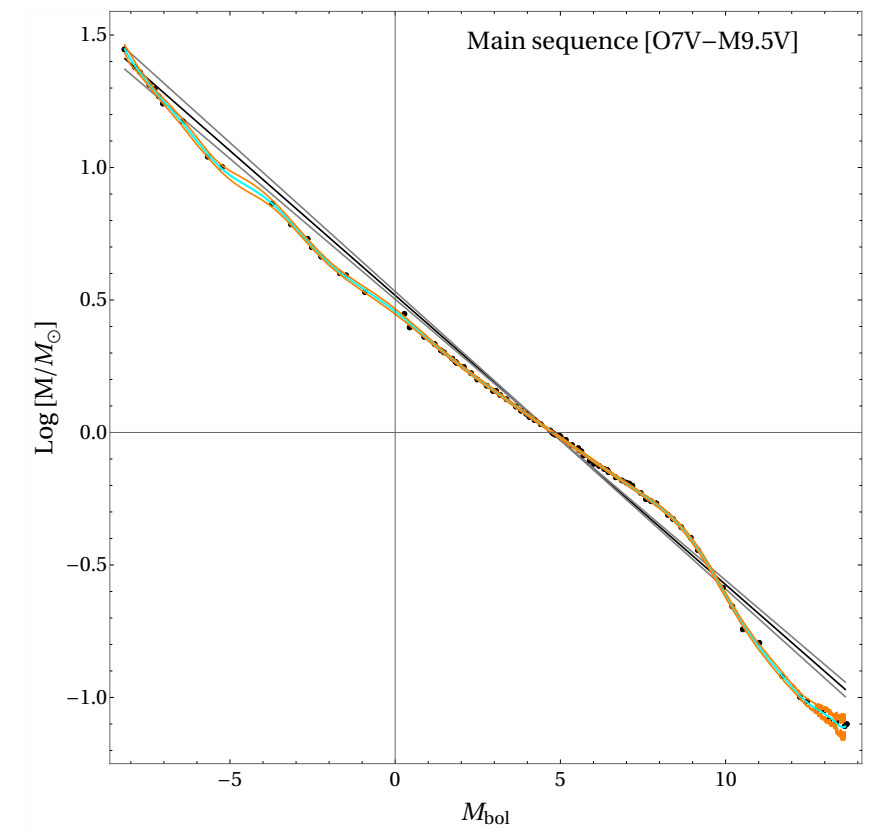

Fig. 1. Black points indicate the data of Pecaut \& Mamajek (2013), updated as of 22 March 2019. The linear mass-luminosity fit as well as the polynomial mass-luminosity fit of degree 20 are shown in black and cyan, respectively. In addition, the $95 \%$ confidence bands for each of them are plotted in grey and orange, respectively.

\subsubsection{A new non-linear MLR}

Given the non-linear shape of the MLR, most investigations have found that any attempt of modelling it with a linear function cannot produce a good-quality fit to observational data through the entire MS. In practice, this problem is usually overcame by adopting piecewise MLRs based on the classical MLR with different parameters depending on the bolometric magnitude range. Nevertheless, piecewise-defined functions would need to be comprised by many pieces to be accurate enough. In addition, such functions may show differentiability and continuity issues that are difficult to deal with from a computational point of view.

Therefore, we propose a new model for the MLR which has been derived taking the above-mentioned data (Pecaut \& Mamajek 2013) into account. This robust non-linear MLR was obtained after evaluating polynomials given in Expression (4) with increasing values of the exponent $n$ up to degree 40. Furthermore, we have seen that polynomials with degrees above a certain value either do not adequately fit some intermediate values or provide overly wide confidence intervals.

A first approach to chose the best precise polynomial would consist in taking that with the highest adjusted coefficient of determination $\left(\bar{R}^{2}\right)$. Nevertheless, we have considered the wellknown Akaike's information criterion (AIC; Akaike 1971) as the fundamental tool for the MLR selection. In particular, considering the large number of dependent variables in comparison with the sample size, we have used the second-order variant $\mathrm{AIC}_{\mathrm{c}}$ (Sugiura 1978). Hence, $\mathrm{AIC}_{\mathrm{c}}$ values are rescaled by an additive constant such that the polynomial with the minimum $\mathrm{AIC}_{\mathrm{c}}$ value has $\Delta_{n}=0$. Formally, $\Delta_{n}=\mathrm{AIC}_{\mathrm{c}, n}-\mathrm{AIC}_{\mathrm{c}, \min }$, with $n$ being the index (degree) of the polynomial. Finally, Akaike weights are calculated according to

$w_{n}=\frac{\mathrm{e}^{-\frac{1}{2} \Delta_{n}}}{\sum_{k=1}^{m} \mathrm{e}^{-\frac{1}{2} \Delta_{k}}}$, 


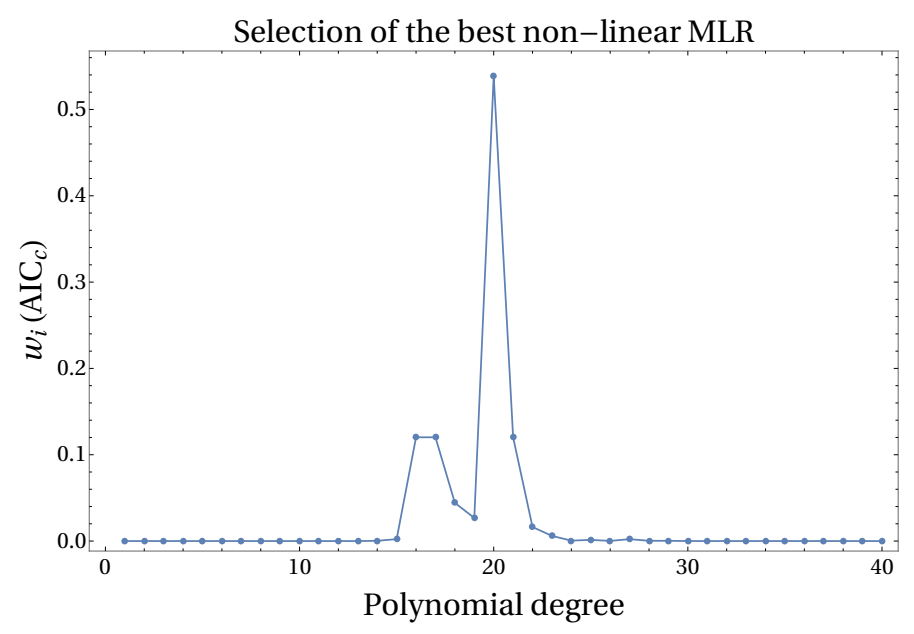

Fig. 2. Akaike weights for the non-linear MLR polynomial models until degree 40 .

where $m$ is the number of considered polynomials. Akaike weight $w_{n}$ may be interpreted as the probability that polynomial $n$ is the best precise model for the sampling situation considered. These weights are plotted for each polynomial of degree $n$ in Fig. 2.

As a result, the best precise fit would be that given by the polynomial of degree $n=20$, which has an adjusted coefficient of determination $\bar{R}^{2}=0.9999$. According to the usual rules of thumb (Burnham \& Anderson 2002), a few polynomials with degrees of around 20 could have a certain level of empirical support. However, that of degree 20 is by far the best precise polynomial, with a probability of $53.9 \%$ (the following best polynomials have only a $12 \%$ probability). From this point of view, this polynomial would be an optimal solution for the problem of the non-linear MLR fit.

The polynomial coefficients and uncertainties are listed in Table 2, whereas the plot with the polynomial fit is shown in Fig. 1. This fit is valid through the entire MS. Indeed, from a numerical point of view, it exhibits a high accuracy because the mean and the standard deviation of the residuals are only of $10^{-11} \mathcal{M}_{\odot}$ and $0.014 \mathcal{M}_{\odot}$, respectively.

\subsection{Interstellar extinction}

In order to estimate the interstellar extinction we use the wellknown exponential extinction law proposed by Parenago (1945),

$A_{\mathrm{V}}(r, b)=\frac{a_{0} \beta}{|\sin b|}\left(1-\mathrm{e}^{-\frac{r|\sin b|}{\beta}}\right)$,

where $r$ is the distance and $b$ the galactic latitude. The parameters $a_{0}$ and $\beta$ are, respectively, the extinction coefficient (i.e. the magnitude of the extinction per parsec to an object located in the galactic plane behind the absorbing layer) and the half-thickness of the dust layer (in parsec). For these parameters we adopt the mean values $a_{0}=1.6 \mathrm{mag} \mathrm{kpc}^{-1}$ and $\beta=11 \pm 2 \mathrm{pc}$ (Sharov 1964).

\section{A colour-dependent model: ANAPAR}

The term model is used to refer jointly to the analytical theory and the MRL fit chosen to perform the calculations. In this way, the model presented in this paper (henceforth referred to as
Table 2. Coefficients of the new non-linear MLR fit.

\begin{tabular}{lc}
\hline \hline Coefficients & Values \\
\hline$c_{0}$ & $(+4.56873 \pm 0.04387) \times 10^{-1}$ \\
$c_{1}$ & $(-9.74625 \pm 0.38883) \times 10^{-2}$ \\
$c_{2}$ & $(-1.06361 \pm 0.32770) \times 10^{-2}$ \\
$c_{3}$ & $(+1.78251 \pm 1.64622) \times 10^{-3}$ \\
$c_{4}$ & $(+2.48845 \pm 0.75888) \times 10^{-3}$ \\
$c_{5}$ & $(-6.44589 \pm 2.56201) \times 10^{-4}$ \\
$c_{6}$ & $(-1.73621 \pm 0.75157) \times 10^{-4}$ \\
$c_{7}$ & $(+6.47989 \pm 2.04487) \times 10^{-5}$ \\
$c_{8}$ & $(+3.94855 \pm 3.65412) \times 10^{-6}$ \\
$c_{9}$ & $(-2.99545 \pm 0.94950) \times 10^{-6}$ \\
$c_{10}$ & $(+6.85835 \pm 9.52516) \times 10^{-8}$ \\
$c_{11}$ & $(+7.17878 \pm 2.57648) \times 10^{-8}$ \\
$c_{12}$ & $(-5.29704 \pm 1.78791) \times 10^{-9}$ \\
$c_{13}$ & $(-8.58617 \pm 3.83261) \times 10^{-10}$ \\
$c_{14}$ & $(+1.07849 \pm 0.32502) \times 10^{-10}$ \\
$c_{15}$ & $(+3.24148 \pm 2.56039) \times 10^{-12}$ \\
$c_{16}$ & $(-9.59859 \pm 3.43232) \times 10^{-13}$ \\
$c_{17}$ & $(+2.50304 \pm 0.75364) \times 10^{-14}$ \\
$c_{18}$ & $(+2.87500 \pm 1.28815) \times 10^{-15}$ \\
$c_{19}$ & $(-1.99425 \pm 0.74121) \times 10^{-16}$ \\
$c_{20}$ & $(+3.71971 \pm 1.30137) \times 10^{-18}$ \\
\hline &
\end{tabular}

Table 3. Data of Luyten 726-8.

\begin{tabular}{lcc}
\hline \hline & $A($ BL Cet) & $B$ (UV Cet) \\
\hline Spectral type & M5.5 V & M6 V \\
$m_{\mathrm{V}}$ & 12.7 & 13.2 \\
\hline$P$ [yr] & $26.284 \pm 0.038$ \\
$a$ [as] & $2.0584 \pm 0.0097$ \\
\hline
\end{tabular}

ANAPAR) comprises the analytical theory described in Sect. 2 together with the non-linear MLR fit defined in Sect. 3.1.2.

\subsection{Example: Luyten 726-8 (WDS 01388-1758)}

This binary system, also known as Gliese 65, comprises two red dwarfs, BL Cet and UV Cet, the latter being, furthermore, the archetype for the class of flare stars. We have chosen this system because there are accurate measurements of the trigonometric parallax, free of any orbital motion influence. However, we also would like to note that the intrinsic variability of both components makes any measurement of their apparent magnitudes somewhat uncertain.

The dynamical parallax obtained by Angelov (1993) in his paper was 385 mas. Using the same astrophysical data, but applying our exact theory, we obtain $380.78 \pm 6.50$ mas. Nevertheless, new calculations with improved orbital elements (Kervella et al. 2016) and apparent magnitudes (see Table 3) yield 388 mas and $380.76 \pm 3.14$ mas, respectively. For the sake of completeness, we also provide new individual masses, $0.119 \pm$ $0.002 \mathcal{M}_{\odot}$ and $0.110 \pm 0.002 \mathcal{M}_{\odot}$, respectively, which exactly match those expected for the spectral types given in Table 3.

Now, these dynamical parallaxes can be compared with the accurate absolute parallax measured by Geyer et al. (1988) using 195 observations covering most of one revolution, $375 \pm 4$ mas. A similar measurement, $372 \pm 8$ mas, was also obtained by 
Table 4. Results of Luyten 726-8.

\begin{tabular}{lccc}
\hline \hline & $\varpi[\mathrm{mas}]$ & $\mathcal{M}_{A}\left[\mathcal{M}_{\odot}\right]$ & $\mathcal{M}_{B}\left[\mathcal{M}_{\odot}\right]$ \\
\hline Dynamical parallaxes & & & \\
\hline Baize \& Romani (1946) & 332 & 0.18 & 0.17 \\
McCluskey \& Kondo (1972) & 311 & 0.22 & 0.20 \\
Docobo \& Andrade (2013) & 299 & 0.24 & 0.23 \\
Andrade (IV: this work, 2019) & $327.9 \pm 2.3$ & $0.185 \pm 0.003$ & $0.174 \pm 0.003$ \\
Angelov (1993) & 385 & 0.11 & 0.11 \\
Angelov (using 2019 data) & 388 & 0.11 & 0.11 \\
Andrade (ANAPAR: this work, 2019) & $380.76 \pm 3.14$ & $0.119 \pm 0.002$ & $0.110 \pm 0.002$ \\
\hline Trigonometric parallaxes & & & \\
\hline Heintz (1987) & $372 \pm 8$ & 0.12 & 0.12 \\
Geyer et al. (1988) & $375 \pm 4$ & 0.101 & 0.099 \\
YPC (van Altena et al. 1995) & $373.70 \pm 2.70$ & - & - \\
Gaia DR2 (Gaia Collaboration 2018) & $371.00 \pm 0.19$ & - & - \\
\hline
\end{tabular}

Table 5. Statistical properties of the synthetic sample.

\begin{tabular}{lcccc}
\hline \hline Parameter & $\bar{x}$ & $\bar{u}(x)$ & $\operatorname{Min}(x)$ & $\operatorname{Max}(x)$ \\
\hline$\varpi$ [mas] & 33.7 & 3.2 & 1.1 & 187.3 \\
$a$ [mas] & 503.2 & 3.2 & 10.1 & 999.8 \\
$\mathcal{M}_{1}\left[\mathcal{M}_{\odot}\right]$ & 11.73 & 0.06 & 0.37 & 21.98 \\
$\mathcal{M}_{2}\left[\mathcal{M}_{\odot}\right]$ & 6.11 & 0.07 & 0.08 & 21.77 \\
\hline
\end{tabular}

Heintz (1987) using 79 nights. Besides that, the weighted trigonometric parallax derived from the two Gaia DR2 measurements is $371.00 \pm 0.19$ mas, whereas that obtained by van Altena et al. (1995) is $373.70 \pm 2.70$ mas. Results are summarised in Table 4.

Therefore, although there may be some uncertainty in the apparent magnitudes, reliable dynamical parallax and masses are obtained. In fact, our dynamical parallax is metrologically consistent (see, e.g., Kacker et al. 2010) with all the absolute trigonometric parallaxes except for that of Gaia DR2, which could however be somewhat affected by the orbital motion.

\subsection{Validation}

\subsubsection{The synthetic sample}

A synthetic sample of $10^{3}$ binary systems has been used to assess the accuracy of the ANAPAR model. This consists of a randomly generated sample of the fundamental parameters $\left(\varpi, a, \mathcal{M}_{1}\right.$, and $\mathcal{M}_{2}$ ), characterized by the statistical properties listed in Table 5, along with the parameters derived from them $\left(P, m_{\mathrm{V}_{1}}\right.$, and $\left.m_{\mathrm{V}_{2}}\right)$.

\subsubsection{Comparison with the previous models}

The reliability of the new model has been analysed considering the synthetic sample along with each of the parallax models summarised in Table 6.

The mean absolute error (MAE) and the root mean squared error (RMSE) of the residuals (differences between the data and the calculated values) are summarised in Table 7 for the dynamical parallax and the total mass. Besides that, scatterplots with the dynamical and the synthetic values for each of these variables are shown in Fig. 3.

In view of these results, we can conclude that although the classical Baize \& Romani (1946) method shows a certain
Table 6. Parallax models: theories and MLR fits to calculate dynamical parallaxes and individual masses.

\begin{tabular}{lclcl}
\hline \hline Model & Theory & Solution & MLR fit & Type \\
\hline BaiRom46 & $(1)$ & Linear & $(2)$ & Linear \\
Ang93 & $(3)$ & Linear & $(3)$ & Non linear \\
ANAPAR & $(4)$ & Exact & $(5)$ & Non linear \\
\hline
\end{tabular}

References. (1) Baize \& Romani (1946); (2) this paper (Sect. 3.1.1); (3) Angelov (1993); (4) this paper (Sect. 2); (5) this paper (Sect. 3.1.2).

Table 7. Mean absolute error and root mean square error of the residuals of the dynamical parallax and the total mass for the synthetic sample of $10^{3}$ systems considering the three parallax models described in Sect. 4.2.2.

\begin{tabular}{lcccc}
\hline \hline & \multicolumn{2}{c}{ Dynamical parallax (mas) } & \multicolumn{2}{c}{ Mass $\left(\mathcal{M}_{\odot}\right)$} \\
\cline { 2 - 5 } Model & MAE & RMSE & MAE & RMSE \\
\hline BaiRom46 & -1.86 & 2.78 & +3.09 & 3.47 \\
Ang93 & +2.93 & 6.98 & -1.70 & 2.87 \\
ANAPAR & +0.02 & 0.39 & +0.10 & 0.40 \\
\hline
\end{tabular}

tendency to underestimate parallaxes, it provides quite reasonable values. This is not true for the total mass, especially in the case of massive systems, as this method yields values well above expectations. Moreover, taking the high dispersion for the mass ratio into account, we should not expect particularly good results for the individual masses either.

Similar conclusions are reached when considering Angelov's approach, using which neither parallaxes nor masses yield reliable values. These anomalies could be mainly due to the condition imposed in the series expansion given in Eq. (11) which completely removes those terms of an order higher than one that account for the difference of mass between the components. This effect can be seen in Fig. 3, where nearly all the points in the dynamical parallax scatterplot of the Ang93 model are spread well above the perfect fit straight line. Regarding masses, the opposite is seen. The Ang93 model tends to overestimate parallaxes and consequently underestimate the masses. Indeed, this effect would be more noticeable the greater the difference in mass between the components. 

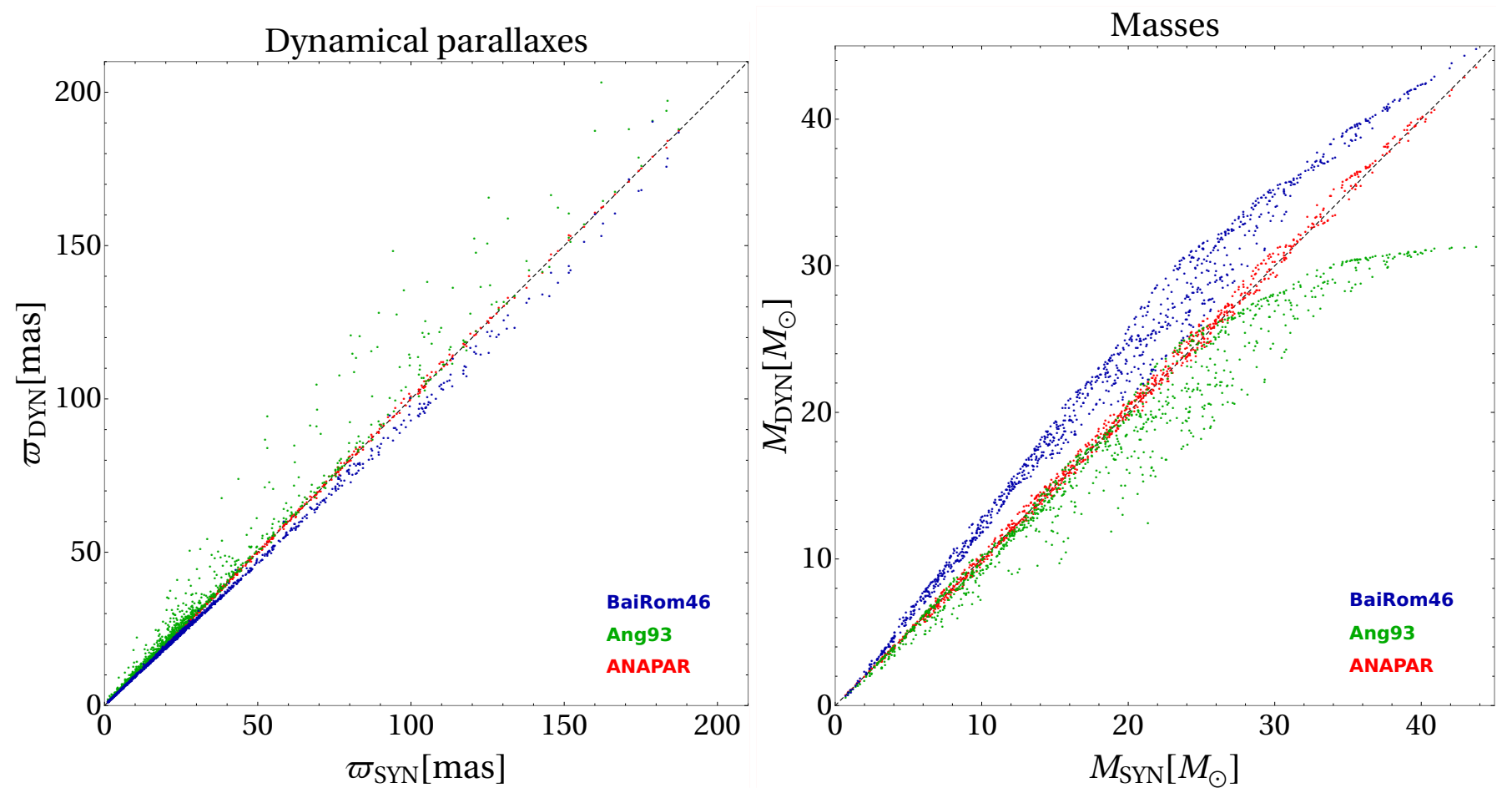

Fig. 3. Scatterplots of dynamical vs. synthetic values (left: dynamical parallaxes, right: total masses) for the synthetic sample of $10^{3}$ systems considering the three parallax models described in Sect. 4.2.2. The black dashed line indicates the perfect fit $(y=x)$.

Quite the contrary, ANAPAR, which computes dynamical parallaxes and individual masses considering the full expression given in Eq. (12) provides results in perfect agreement with data for both dynamical parallaxes and masses. Thus, MAE and RMSE of the ANAPAR residuals yield by far the lowest values for all models (see Table 7).

\section{Application}

\subsection{The $V B+S B 2$ set}

Dynamical parallaxes and individual masses calculated by means of the new model presented in this paper have been compared with trigonometric and orbital parallaxes, and with masses obtained from combined astrometry and spectroscopy data, respectively. This comparison has been carried out considering the set of MS VB+SB2 systems with visual orbits that have been graded 1 (definitive) in the ORB6. There are 19 systems in all, some of them with simultaneous adjustment of the visual and the spectroscopic data.

Unless otherwise indicated, visual orbital elements were taken from the ORB6 and apparent magnitudes from the HIPPARCOS and Tycho Catalogues (ESA 1997) or, if not available, from the ORB6. Likewise, radial velocities needed to calculate the orbital parallaxes were taken from the $S_{B^{9}}$ : ninth catalogue of spectroscopic binary orbits (hereafter, SB9; Pourbaix et al. 2004).

Orbital and dynamical parallaxes for all the models are listed in Table 8. For the sake of completeness, trigonometric parallaxes taken from the general catalogue of trigonometric [stellar] parallaxes (van Altena et al. 1995), from second reduction of HIPPARCos (hereafter, HIP2; van Leeuwen 2007), and from Gaia DR2 (hereafter, GDR2; Gaia Collaboration 2018) are also listed (we note that in one case, STF 2173 AB, the two GDR2 values available for the same system have been combined into a weighted mean parallax).
Nevertheless, we would like to point out that we should be careful with any direct comparison because of possible uncorrected orbital motions in astrometric data. Specifically, it is worth mentioning that current Gaia DR2 parallaxes do not take orbital motion into account and, as a consequence, these should not be considered as the most accurate values (e.g., KUI $37 \mathrm{AB}$ and HO 276, see below). On the contrary, dynamical and orbital parallaxes are often the most reliable sources. Another trustworthy source is that of trigonometric parallaxes of visual binaries corrected by their orbital motions, as is the case for the HIPPARCOS measurements processed by Söderhjelm (1999). Likely, Gaia DR3, which is anticipated to take place during the second half of 2021, will provide unbiased parallaxes as well.

We note that the ANAPAR model applied to the $\mathrm{VB}+\mathrm{SB} 2$ set leads to dynamical parallaxes in agreement with those derived from astrometric and spectroscopic observations. With respect to the derived masses using this method, we obtain individual dynamical masses in reasonable agreement with both orbital and spectral masses, as you can see in Table 8. Discrepancies in some particular cases are discussed below. Estimated spectral types are also listed in Table 8.

Uncertainties in both dynamical parallaxes and masses have been calculated using Monte Carlo simulations according to the JCGM:101 (2008). This approach involves the repeated simulation of samples within the probability density functions (PDFs) of the input data (period, semimajor axis, and apparent magnitudes). As usual, we assume that each of these variables, $X_{i}$, are normally distributed, thus $X_{i} \sim \mathcal{N}\left(\mu_{i}, \sigma_{i}^{2}\right)$, where $\mu_{i}$ is the expectation and $\sigma_{i}$ the standard deviation of each of them. The next step is to run as many simulations as possible for each binary system. A general rule of thumb is to use $10^{4}$ simulations, since this number of simulations usually leads to stable outcomes in the resulting distributions. At a later stage, it is necessary to plug each of the simulation results into the equations of the model in order to obtain the distributions of the results (parallaxes 
Table 8. Parallaxes, individual masses, and spectral types for the VB+SB2 systems.

\begin{tabular}{|c|c|c|c|c|c|c|c|c|c|}
\hline \multirow[b]{2}{*}{$\begin{array}{l}\text { WDS(2000) } \\
\text { Name }\end{array}$} & \multicolumn{4}{|c|}{ Trigonometric and orbital parallaxes } & \multicolumn{5}{|c|}{ Dynamical model [ANAPAR, this work] } \\
\hline & $\begin{array}{l}\varpi_{\mathrm{trig}}^{\mathrm{ALT}} \\
{[\mathrm{mas}]}\end{array}$ & $\begin{array}{l}\varpi_{\text {trig }}^{\mathrm{HIP}} \\
{[\mathrm{mas}]}\end{array}$ & $\begin{array}{l}\varpi_{\text {trig }}^{\mathrm{GDR} 2} \\
{[\mathrm{mas}]}\end{array}$ & $\begin{array}{c}\varpi_{\text {orb }} \\
{[\mathrm{mas}]}\end{array}$ & $\begin{array}{c}\varpi_{\text {dyn }} \\
{[\text { mas }]}\end{array}$ & $\begin{array}{c}\mathcal{M}_{\text {dyn }, 1} \\
{\left[\mathcal{M}_{\odot}\right]}\end{array}$ & $\begin{array}{c}\mathcal{M}_{\mathrm{dyn}, 2} \\
{\left[\mathcal{M}_{\odot}\right]}\end{array}$ & $\begin{array}{l}\mathrm{Sp}_{1}[\mathrm{~V}] \\
\mathrm{Sp}_{2}[\mathrm{~V}]\end{array}$ & Ref. \\
\hline $00352-0336$ & 61.5 & 47.05 & - & 45.61 & 52.54 & 1.137 & 0.896 & F9 & $(1 ; 2)$ \\
\hline $\mathrm{HO} 212 \mathrm{AB}^{(\dagger)}$ & \pm 4.5 & \pm 0.67 & & \pm 1.43 & \pm 0.66 & \pm 0.007 & \pm 0.011 & G9 & \\
\hline $00373-2446$ & - & 64.93 & - & 66.94 & 65.12 & 0.915 & 0.864 & G9 & $(3 ; 4)$ \\
\hline BU 395 & & \pm 1.85 & & \pm 2.16 & \pm 0.80 & \pm 0.005 & \pm 0.005 & K0.5 & \\
\hline $02278+0426$ & 62.2 & 58.33 & - & 54.03 & 61.47 & 0.594 & 0.579 & K8 & $(5 ; 6)$ \\
\hline A 2329 & \pm 4.1 & \pm 1.08 & & \pm 2.59 & \pm 1.73 & \pm 0.012 & \pm 0.012 & K8 & \\
\hline 02396-1152 & 61.8 & 46.55 & 42.4864 & 35.07 & 41.33 & 1.361 & 1.060 & $\mathrm{~F} 4$ & $(7 ; 4)$ \\
\hline FIN 312 & \pm 6.3 & \pm 2.53 & \pm 0.7591 & \pm 1.00 & \pm 0.68 & \pm 0.042 & \pm 0.095 & G1 & \\
\hline $02442-2530$ & - & 24.20 & 22.4133 & 22.25 & 21.73 & 1.129 & 1.063 & F9 & (8) \\
\hline FIN $379 \mathrm{Aa}, \mathrm{Ab}$ & & \pm 1.16 & \pm 0.1760 & \pm 0.35 & \pm 0.19 & \pm 0.024 & \pm 0.023 & G1 & \\
\hline $03082+4057$ & 38.3 & 36.27 & - & 34.98 & 35.99 & 3.408 & 1.635 & B8 & $(9 ; 10)$ \\
\hline LAB $2 \mathrm{Aa}, \mathrm{Ab}^{(\dagger)}$ & \pm 3.4 & \pm 1.40 & & \pm 1.02 & \pm 0.37 & \pm 0.144 & \pm 0.037 & A9 & \\
\hline $04256+1556$ & 37.7 & 20.87 & 18.9472 & 23.77 & 23.46 & 1.218 & 1.057 & F7 & $(11 ; 12)$ \\
\hline FIN $342 \mathrm{Aa}, \mathrm{Ab}$ & \pm 15.1 & \pm 0.72 & \pm 0.2819 & \pm 0.52 & \pm 0.39 & \pm 0.038 & \pm 0.060 & G1 & \\
\hline $07518-1354$ & 60.2 & 60.59 & - & 64.23 & 60.46 & 1.061 & 0.900 & G1 & $(13 ; 4)$ \\
\hline BU 101 & \pm 3.1 & \pm 0.59 & & \pm 2.35 & \pm 0.28 & \pm 0.002 & \pm 0.002 & G9 & \\
\hline $09006+4147$ & 66.1 & 62.23 & 71.1893 & 56.29 & 62.70 & 1.396 & 0.902 & F4 & $(14 ; 6)$ \\
\hline KUI $37 \mathrm{AB}$ & \pm 6.5 & \pm 0.68 & \pm 1.4693 & \pm 1.06 & \pm 0.09 & \pm 0.002 & \pm 0.005 & G9 & \\
\hline $09123+1500$ & 47.7 & 49.11 & 49.1493 & 51.01 & 50.22 & 0.865 & 0.865 & K0 & $(15 ; 16)$ \\
\hline FIN $347 \mathrm{Aa}, \mathrm{Ab}$ & \pm 5.1 & \pm 0.54 & \pm 0.3467 & \pm 0.84 & \pm 0.67 & \pm 0.017 & \pm 0.017 & K0 & \\
\hline $15232+3017$ & 59.8 & 55.98 & - & 54.62 & 56.02 & 1.081 & 1.023 & G0 & $(14 ; 4)$ \\
\hline STF 1937 AB & \pm 5.8 & \pm 0.78 & & \pm 0.93 & \pm 0.03 & \pm 0.001 & \pm 0.001 & $\mathrm{G} 2$ & \\
\hline $16555-0820$ & 154.8 & 161.41 & - & 172.36 & 177.42 & 0.372 & 0.366 & M3 & $(11 ; 17)$ \\
\hline KUI $75 \mathrm{AB}^{(\dagger)}$ & \pm 0.6 & \pm 5.64 & & \pm 9.43 & \pm 3.48 & \pm 0.015 & \pm 0.015 & M3 & \\
\hline $17304-0104$ & 55.3 & 61.19 & 59.1255 & 61.54 & 61.13 & 0.963 & 0.951 & G7 & $(18 ; 4)$ \\
\hline STF $2173 \mathrm{AB}$ & \pm 4.4 & \pm 0.68 & \pm 0.6405 & \pm 1.20 & \pm 0.75 & \pm 0.005 & \pm 0.005 & G7 & \\
\hline $18055+0230$ & 199.7 & 196.72 & - & 192.15 & 199.22 & 0.848 & 0.653 & $\mathrm{~K} 1$ & $(19 ; 4)$ \\
\hline STF $2272 \mathrm{AB}$ & \pm 3.4 & \pm 0.83 & & \pm 4.36 & \pm 0.41 & \pm 0.001 & \pm 0.004 & K6 & \\
\hline $18211+7244$ & 128.8 & 124.11 & - & 123.43 & 118.29 & 1.163 & 0.808 & F8 & $(20 ; 4)$ \\
\hline LAB $5 \mathrm{Aa}, \mathrm{Ab}$ & \pm 2.9 & \pm 0.87 & & \pm 1.97 & \pm 1.38 & \pm 0.026 & \pm 0.015 & $\mathrm{~K} 1.5$ & \\
\hline $19091+3436$ & 24.7 & 19.11 & 20.2306 & 27.87 & 24.55 & 1.233 & 0.940 & F6 & $(21 ; 22)$ \\
\hline $\mathrm{CHR} 84 \mathrm{Aa}, \mathrm{Ab}$ & \pm 11.1 & \pm 2.35 & \pm 0.3846 & \pm 1.40 & \pm 1.20 & \pm 0.027 & \pm 0.021 & G8 & \\
\hline $19311+5835$ & 49.3 & 58.96 & 54.8715 & 56.10 & 57.21 & 0.850 & 0.701 & K1 & (23) \\
\hline MCA 56 & \pm 5.0 & \pm 0.65 & 0.2400 & \pm 0.88 & \pm 0.99 & \pm 0.023 & \pm 0.049 & $\mathrm{~K} 4.5$ & \\
\hline 19598-0957 & 39.8 & 45.04 & 46.9328 & 40.21 & 42.29 & 1.105 & 0.832 & F9.5 & $(24 ; 4)$ \\
\hline HO 276 & \pm 7.9 & \pm 0.99 & \pm 0.9290 & \pm 5.74 & \pm 0.45 & \pm 0.009 & \pm 0.020 & K1.5 & \\
\hline $21145+1000$ & 54.5 & 54.09 & 53.5882 & 54.76 & 54.21 & 1.214 & 1.193 & F7 & $(25 ; 4)$ \\
\hline STT 535 AB & \pm 0.9 & \pm 0.66 & \pm 0.7292 & \pm 0.48 & \pm 0.12 & \pm 0.008 & \pm 0.012 & F8 & \\
\hline
\end{tabular}

Notes. Trigonometric parallaxes given by van Altena et al. (1995), HIP2 (van Leeuwen 2007), and GDR2 (Gaia Collaboration 2018) are listed in Cols. 2-4, respectively, whereas orbital parallaxes are listed in Col. 5. On the other hand, dynamical parallaxes and individual masses obtained from the exact method presented in Sect. 2 using the non-linear MLR defined in Sect. 3.1.2, together with the suggested spectral types estimated from the Pecaut \& Mamajek (2013) data, are listed in Cols. 6-9, respectively. Finally, references for the orbital elements are listed in the last column. ${ }^{(\dagger)} \mathrm{HO} 2012 \mathrm{AB}$ : A is an SB1 star/LAB 2 Aa,Ab: Aa is an SB2 star/KUI 75 AB: B is an SB2 star. See Table 9 for more details.

References. (1) Mason \& Hartkopf (2005); (2) Duquennoy \& Mayor (1991); (3) Hartkopf \& Mason (2010); (4) Pourbaix (2000); (5) Andrade (2007); (6) Agati et al. (2015); (7) Docobo \& Andrade (2013); (8) Tokovinin (2016); (9) Baron et al. (2012); (10) Hill et al. (1971); (11) Söderhjelm (1999); (12) Griffin (2012); (13) Tokovinin (2012); (14) Muterspaugh et al. (2010); (15) Mason \& Hartkopf (2012); (16) Halbwachs et al. (2018); (17) Mazeh et al. (2001); (18) Heintz (1994); (19) Eggenberger et al. (2008); (20) Farrington et al. (2010); (21) Farrington et al. (2014); (22) Tokovinin et al. (2000); (23) Kiefer et al. (2018); (24) Tokovinin (2017); (25) Muterspaugh et al. (2008).

and masses). As it is customary to do, their standard deviations would be taken as estimates of the corresponding uncertainties.

Finally, we would like to note that dynamical parallax theories may suffer from a lack of accuracy when there are more than two components in the system. In these cases we can use the method of Docobo \& Andrade (2006), henceforth referred to as DA2006, to analyse triple stellar systems that include a visual double star wherein one of the components is a spectroscopic 
binary. This method has been applied in three cases (HO $212 \mathrm{AB}$, $\mathrm{LAB} 2 \mathrm{Aa}, \mathrm{Ab}$, and KUI $75 \mathrm{AB}$ ) using updated data. As a result, the most probable values of the inclination of the spectroscopic binary, the angular separation between its components, their masses, as well as their spectral types are also provided (see Table 9). We note that these, calculated specifically for systems known to be triple, could be more accurate than those shown in Table 8.

\subsection{Comments on the individual systems}

00352-0336 (HO $212 A B$ ). The variable star 13 Cet is a triple star (A is itself a 2.1 days SB1 star) which belongs to the Hyades moving group (vB 77). Its total mass, derived from its visual orbital elements and from the HIP2 parallax, is about $2.8 \mathcal{M}_{\odot}$. Thus, there is an excess of mass with respect to both our preliminary dynamical mass $\left(2.033 \pm 0.013 \mathcal{M}_{\odot}\right)$ and that $\left(2.20 \mathcal{M}_{\odot}\right)$ estimated considering its binary spectral classification, F7 V + G4 V (Strassmeier et al. 1993).

A reasonable hypothesis is that this discrepancy is probably due to the third inner companion $\mathrm{Ab}$, likely a $\mathrm{K}$ dwarf, which could also explain the relatively large difference between the inferred dynamical parallax and the trigonometric parallax. Nevertheless, the application of the DA2006 method allows a parallax of $47.34 \pm 2.18$ mas to be derived, which closely matches that estimated by Söderhjelm (1999) using the intermediate transit data of HIPPARCos, $47.73 \pm 1.21$ mas. Individual masses and spectral types for the three components, together with additional orbital parameters for the spectroscopic orbit, are summarised in Table 9.

00373-2446 (BU 395). This binary system could comprise, according to Houk \& Smith-Moore (1988) and Gray et al. (2006), a G7 V star plus a K1 V companion. As a consequence, its total mass would be around $1.8 \mathcal{M}_{\odot}$, in complete agreement with that derived from the orbital elements $\left(1.79 \pm 0.16 \mathcal{M}_{\odot}\right)$ and with that calculated using our dynamical model $\left(1.779 \pm 0.007 \mathcal{M}_{\odot}\right)$. A reliable dynamical parallax, very close to that measured by HIP2, is also provided.

02278+0426 (A 2329). This K7 V system (Andrade 2007) probably comprises a pair of mid-late K dwarfs. Available measurements of the trigonometric parallax from van Altena et al. (1995) and from HIP2 (Gaia is not available) provide a total mass of $1.13 \pm 0.24 \mathcal{M}_{\odot}$ and $1.37 \pm 0.12 \mathcal{M}_{\odot}$, respectively. Therefore, the total mass calculated in this paper $\left(1.173 \pm 0.017 \mathcal{M}_{\odot}\right)$ lies between the two. On the other hand, individual masses (see Table 8) agree with the spectral type. Moreover, fractional mass $\left(\mathcal{M}_{2} /\left(\mathcal{M}_{1}+\mathcal{M}_{2}\right)=0.494 \pm 0.007\right)$ agrees well with that of 0.47 given by Agati et al. (2015). Nonetheless, the individual masses that these latter authors derived from their orbital elements $\left(0.95 \mathcal{M}_{\odot}\right.$ and $0.85 \mathcal{M}_{\odot}$, respectively) seem to be too large in comparison with those expected taking the spectral type into account.

This is a good example of a low-accuracy determination of the orbital parallax due to minor systematic errors in the orbital elements, likely in the radial velocities.

02396-1152 (FIN 312). The $\epsilon$ Cet system is a linewidth spectroscopic binary (LWSB), with spectral type F4 V (Abt 2009), that shows a large discrepancy between its orbital mass derived from its orbital parallax using Kepler's third law $\left(3.962 \pm 0.251 \mathcal{M}_{\odot}\right)$ and that derived from the dynamical model $\left(2.421 \pm 0.104 \mathcal{M}_{\odot}\right)$. Nevertheless, the latter matches very well with that expected for the combined spectral type for a binary
Table 9. Derived quantities for the $\mathrm{VB}+\mathrm{SB} 2$ systems with spectroscopic subcomponents.

\begin{tabular}{lccc}
\hline \hline WDS(2000) & $\mathcal{M}_{\text {dyn,1 } 1}\left[\mathcal{M}_{\odot}\right]$ & $\mathrm{Sp}_{1}$ & $\varpi[\mathrm{mas}]$ \\
Name & $\mathcal{M}_{\text {dyn,2 }}\left[\mathcal{M}_{\odot}\right]$ & $\mathrm{Sp}_{2}$ & $a_{12}[\mathrm{mas}]$ \\
Components & $\mathcal{M}_{\left.\text {dyn,3 } 3 \mathcal{M}_{\odot}\right]}$ & $\mathrm{Sp}_{3}$ & $i_{12}[\mathrm{deg}]$ \\
\hline 00352-0336 & $1.18 \pm 0.09$ & $\mathrm{~F} 6 \mathrm{~V}$ & $47.34 \pm 2.18$ \\
HO 212 AB & $0.70 \pm 0.09$ & $\mathrm{~K} 3.5 \mathrm{~V}$ & $1.86 \pm 0.38$ \\
(Aa,Ab),B & $0.90 \pm 0.09$ & $\mathrm{G} 4 \mathrm{~V}$ & $35.9 \pm 7.3$ \\
\hline 03082+4057 & $3.65 \pm 0.12$ & $\mathrm{~B} 8 \mathrm{~V}$ & $33.53 \pm 0.31$ \\
LAB 2 Aa,Ab & $1.07 \pm 0.07$ & $\mathrm{~K} 2 \mathrm{IV}$ & $2.22 \pm 0.78$ \\
(Aa1,Aa2),Ab & $1.52 \pm 0.10$ & $\mathrm{~F} 1 \mathrm{~V}$ & $78 \pm 66$ \\
\hline 16555-0820 & $0.46 \pm 0.09$ & $\mathrm{M} 3 \mathrm{~V}$ & $146.38 \pm 8.63$ \\
KUI 75 AB & $0.43 \pm 0.09$ & $\mathrm{M} 3.5 \mathrm{~V}$ & $5.6 \pm 1.3$ \\
$\mathrm{~A},(\mathrm{Ba}, \mathrm{Bb})$ & $0.43 \pm 0.09$ & $\mathrm{M} 3.5 \mathrm{~V}$ & $15.8 \pm 3.6$ \\
\hline
\end{tabular}

Notes. Derivation of the dynamical parallaxes and individual masses summarised in this table was carried out using the method of Docobo \& Andrade (2006) and therefore, as usual, a linear MLR.

with a difference of magnitudes $\Delta m=1.29 \pm 0.46$ (about $1.4 \mathcal{M}_{\odot}$ for a F2 and $1.1 \mathcal{M}_{\odot}$ for a F9). On the other hand, a total mass of $2.23 \pm 0.12 \mathcal{M}_{\odot}$ is also obtained from the visual orbital elements along with the GDR2 parallax.

Anyhow, individual masses derived from the dynamical model $\left(1.361 \pm 0.042 \mathcal{M}_{\odot}\right.$ and $\left.1.060 \pm 0.095 \mathcal{M}_{\odot}\right)$ corroborate those of Docobo \& Andrade (2013), $1.37 \pm 0.09 \mathcal{M}_{\odot}$ and $1.03 \pm 0.08 \mathcal{M}_{\odot}$. All of these mass values are in agreement with those calculated by Söderhjelm (1999): $1.41 \pm 0.15 \mathcal{M}_{\odot}$ and $1.19 \pm 0.13 \mathcal{M}_{\odot}$. The parallax $(40.59 \pm 1.31$ mas $)$ of this latter author is also in good agreement with our dynamical parallax $(41.33 \pm 0.68$ mas $)$.

The recent GDR2 parallax would confirm that Martin \& Mignard (1998), using astrometric data of HIPPARCos, underestimated the value of the parallax $(26.22 \pm 1.08$ mas $)$, which in turn resulted in an excessively large mass of the first companion $\left(1.886 \pm 0.17 \mathcal{M}_{\odot}\right)$. Finally, we would like to point out that the orbital parallax shown in Table 8 was first given in Docobo \& Andrade (2015).

02442-2530 (FIN $379 A a, A b)$. The various parallaxes obtained for this G3 V binary system (Houk \& Smith-Moore 1988) are in good agreement. In particular, dynamical and GDR2 parallaxes are very similar, but somewhat lower $(8 \%)$ than that given by HIP2. Total mass derived in this paper $\left(2.192 \pm 0.033 \mathcal{M}_{\odot}\right)$ is slightly larger than that $(2.03 \pm 0.06)$ obtained by Tokovinin (2016), the latter being closer to that expected for a pair of early $\mathrm{G}$ stars. Nevertheless, similar values are obtained for the mass ratios, $0.942 \pm 0.029$ (this work) and $0.933 \pm 0.059$ (Tokovinin 2016).

$03082+4057$ (LAB $2 A a, A b)$. The $\beta$ Per system, better known as Algol and whose main component has been classified as B8 V (Gray et al. 2003), is a triple system (Aa is itself a 2.9 days SB2 star). The Aa 2 component is a subgiant of spectral type K2 IV and Ab seems to be a F1 V star (Richards 1993). Components Aa1, Aa2, and Ab would have $3.7 \pm 0.2 \mathcal{M}_{\odot}, 0.8 \pm 0.1 \mathcal{M}_{\odot}$, and $1.5 \pm 0.1 \mathcal{M}_{\odot}$, respectively, according to Zavala et al. (2010). On the other hand, Baron et al. (2012) estimated 3.17 $\pm 0.21 \mathcal{M}_{\odot}$, $0.70 \pm 0.08 \mathcal{M}_{\odot}$, and $1.76 \pm 0.15 \mathcal{M}_{\odot}$, respectively.

Furthermore, the application of DA2006 methodology provides a parallax $(33.53 \pm 0.31$ mas $)$ closer to that $(34.7 \pm 0.6$ mas) obtained by Zavala et al. (2010). On the other hand, the dynamical parallax derived from our model $(35.99 \pm 0.37$ mas $)$ 
is very similar to that provided by HIP2 $(36.27 \pm 1.40$ mas $)$. Individual masses and spectral types for the three components, together with additional orbital parameters for the spectroscopic orbit, are summarised in Table 9.

$04256+1556$ (FIN $342 A a, A b)$. The 70 Tau system is a F7 V (Gray et al. 2001) system which belongs to the Hyades moving group (vB 57). In this case the new spectroscopic solution determined by Griffin (2012) has been used to calculate the orbital parallax instead of that of the SB9. Dynamical parallax $(23.46 \pm 0.39$ mas $)$ is in agreement with orbital parallax $(23.77 \pm 0.52$ mas), but not with the trigonometric measurements; particularly that of GDR2 (18.9472 \pm 0.2819 mas) is significantly lower than those. Nevertheless, dynamical mass ratio $(q=0.868 \pm 0.056)$ is consistent with the spectral type and the difference of magnitudes $(\Delta m=0.74 \pm 0.31)$.

07518-1354 (BU 101). The 9 Pup system is a G0 V system (Gray et al. 2006) with dynamical $(60.46 \pm 0.28$ mas) and trigonometric parallaxes $(60.59 \pm 0.59$ mas, HIP2) in complete agreement. Dynamical masses also agree with those expected for the spectral type and the difference of magnitudes $(\Delta m=$ $0.90 \pm 0.01)$

$09006+4147$ (KUI 37 AB). The $10 \mathrm{UMa}$ system is a F3 V + G5 V system (Muterspaugh et al. 2010) whose dynamical parallax $(62.70 \pm 0.09$ mas $)$ is in perfect agreement with that given by HIP2 $(62.23 \pm 0.68$ mas), but not with that of GDR2 $(71.1893 \pm 1.4693 \mathrm{mas})$, whose biased value is likely due to the uncorrected orbital motion.

Nevertheless, individual dynamical masses $(1.397 \pm 0.002$ $\mathcal{M}_{\odot}$ are $\left.0.902 \pm 0.005 \mathcal{M}_{\odot}\right)$ are consistent with the previous estimations of Söderhjelm (1999) of $1.37 \pm 0.08 \mathcal{M}_{\odot}$ and $1.04 \pm 0.06$ $\mathcal{M}_{\odot}$, as well as with spectral types. In view of these results, we suggest that the GDR2 parallax could be overestimated by roughly $14 \%$.

09123+1500 (FIN 347 Aa,Ab). Dynamical parallax (50.22 \pm 0.67 mas) calculated for the $\pi^{1}$ Cnc system, classified as G9 V (Gray et al. 2003), agrees well with trigonometric parallaxes (49.1493 \pm 0.3467 mas, GDR2). Interestingly enough, the measurement provided by GDR1 (Gaia Collaboration 2016), $50.230 \pm 0.340$, matches almost perfectly with our dynamical parallax.

Reliable individual masses are obtained $\left(0.865 \pm 0.017 \mathcal{M}_{\odot}\right.$ for each component), similar to those previously derived by Pourbaix (2000), $0.890 \pm 0.029 \mathcal{M}_{\odot}$ and $0.850 \pm 0.025 \mathcal{M}_{\odot}$, respectively.

15232+3017 (STF 1937 AB). Components of $\eta \mathrm{CrB}$ have been classified G2 V + G2 V (Struve \& Franklin 1955), but later Bidelman (1985) estimated a system spectral type F9 V. Our dynamical parallax $(56.02 \pm 0.03$ mas $)$ is in perfect agreement with the trigonometric value of HIP2 (Gaia not available), $55.98 \pm 0.78$ mas, as well as with individual masses for a $\mathrm{G} 0 \mathrm{~V}+\mathrm{G} 2 \mathrm{~V}$ system $\left(1.081 \pm 0.001 \mathcal{M}_{\odot}\right.$ and $\left.1.023 \pm 0.001 \mathcal{M}_{\odot}\right)$.

16555-0820 (KUI 75 AB). Gliese 644 comprises three red dwarfs, $\mathrm{A}, \mathrm{Ba}$, and $\mathrm{Bb}$, all of them visible in the spectra. Its spectral type is M3.5 V (Joy \& Abt 1974) and the individual masses are $0.4155 \pm 0.0057 \mathcal{M}_{\odot}, 0.3466 \pm 0.0047 \mathcal{M}_{\odot}$, and $0.3143 \pm 0.0040 \mathcal{M}_{\odot}$, according to Ségransan et al. (2000), or $0.410 \pm 0.028 \mathcal{M}_{\odot}, 0.336 \pm 0.016 \mathcal{M}_{\odot}$, and $0.304 \pm 0.014 \mathcal{M}_{\odot}$, respectively, according to Mazeh et al. (2001). The former authors also provide spectral types M3 V, M3.5 V, and M3.5 V, for the respective components.
Nevertheless, these masses were derived adopting the ground-based value of the parallax given by van Altena et al. (1995), $154.8 \pm 0.6$ mas, since they claim that it is the most precise. On the other hand, the uncertainty in the trigonometric parallax given by HIP2 (161.41 \pm 5.64 mas) is large enough for these measurements to be compatible with each other. Alternatively, discrepancies with dynamical masses (and maybe with dynamical parallax as well) could be easily explained by the presence of a third companion.

The application of the DA2006 methodology provides a new parallax (146.38 \pm 8.63 mas), individual masses, and spectral types for the three components, together with additional orbital parameters for the spectroscopic orbit (see Table 9).

However, apart from the parallaxes given by van Altena et al. (1995), $154.8 \pm 0.6$ mas, and Söderhjelm (1999), $155.63 \pm$ 1.81 mas, none of the other values come close to the reliable measurements (free of any orbital motion influence) given by GDR2 for the distant physical companions C (Gliese 643) and $F$ (Gliese $644 \mathrm{C}$ ), namely $153.9189 \pm 0.1310$ mas and $153.8139 \pm 0.1148$ mas, respectively.

17304-0104 (STF $2173 A B$ ). The dynamical parallax $(61.13 \pm 0.75$ mas) for this G9 IV-V binary system (Keenan \& McNeil 1989) is in complete agreement with that measured by HIP2 (61.19 \pm 0.68 mas). Individual masses of $0.963 \pm 0.005 \mathcal{M}_{\odot}$ and $0.951 \pm 0.005 \mathcal{M}_{\odot}$ are also consistent with those of Pourbaix (2000), $0.980 \pm 0.052 \mathcal{M}_{\odot}$ and $0.900 \pm 0.045 \mathcal{M}_{\odot}$.

$18055+0230$ (STF $2272 A B$ ). The 70 Oph is a K0 V system (Keenan \& McNeil 1989) placed at only 5 pc. Its dynamical parallax (199.22 \pm 0.41 mas) agrees very well with that of van Altena et al. (1995), $199.7 \pm 3.4$ mas.

The dynamical masses for each component, $0.848 \pm$ $0.001 \mathcal{M}_{\odot}$ and $0.653 \pm 0.004 \mathcal{M}_{\odot}$, are also consistent with the spectral type and the difference of magnitudes $\Delta m=1.84 \pm 0.04$.

$18211+7244$ (LAB $5 A a, A b)$. The $\chi$ Dra system $\mathrm{A}$ is a F7 V system (Gray et al. 2001) which probably consists of a F6V and a late-G/early-K dwarf pair with a difference of about 2 magnitudes in apparent brightness. Our dynamical masses $\left(1.163 \pm 0.026 \mathcal{M}_{\odot}\right.$ and $\left.0.808 \pm 0.015 \mathcal{M}_{\odot}\right)$ are in better agreement with the masses expected for these spectral types than those derived by Farrington et al. (2010) using the parallax given by HIP2 $\left(0.96 \pm 0.03 \mathcal{M}_{\odot}\right.$ and $0.75 \pm 0.03$ $\mathcal{M}_{\odot}$ ). A reasonable explanation for this discrepancy may be a hypothetical overestimation of the HIP2 trigonometric parallax (124.11 \pm 0.87 mas) as a result of the orbital motion of this shortperiod binary (about 280 days). Indeed, our dynamical parallax yields $118.29 \pm 1.38$ mas.

19091+3436 (CHR $84 A a, A b)$. Individual spectral types of this binary system are G1 V + K1 V (Tokovinin et al. 2000). However, these latter authors pointed out that the only actual MK spectral classification is G5 V (Harlan 1974). In this case, the new spectroscopic solution determined by Farrington et al. (2014) has been used to calculate the orbital parallax instead of that given in the SB9. These latter authors obtained very small masses $\left(0.802 \pm 0.055 \mathcal{M}_{\odot}\right.$ and $\left.0.622 \pm 0.053 \mathcal{M}_{\odot}\right)$ considering the individual spectral types. On the contrary, we obtain somewhat large dynamical masses $\left(1.234 \pm 0.027 \mathcal{M}_{\odot}\right.$ and $0.942 \pm 0.021 \mathcal{M}_{\odot}$ ). In any case, expected masses derived from the individual spectral types (roughly 1 and $0.8 \mathcal{M}_{\odot}$, respectively) should be treated with caution. On the other hand, our dynamical parallax $(24.55 \pm 1.20$ mas $)$ matches well with that of van Altena et al. (1995), 24.7 \pm 11.1 mas, in spite of its huge uncertainty, although values are significantly larger 
than the measurements of HIP2 and GDR2, $19.11 \pm 2.35$ and $20.2306 \pm 0.3846$, respectively.

19311+5835 (MCA 56). The difference in magnitude between the components of this K2 V system (Keenan \& McNeil 1989) is $\Delta m=1.388 \pm 0.515$. The system comprises a pair of $\mathrm{K}$ dwarfs (likely being $\mathrm{K} 1+\mathrm{K} 5$ ). Individual masses for both components $\left(0.833 \pm 0.031 \mathcal{M}_{\odot}\right.$ and $\left.0.812 \pm 0.030 \mathcal{M}_{\odot}\right)$ were recently obtained by Kiefer et al. (2018). However, dynamical masses derived in this paper $\left(0.850 \pm 0.023 \mathcal{M}_{\odot}\right.$ and $0.701 \pm 0.049 \mathcal{M}_{\odot}$ ) seem to be in much better agreement with those expected for the spectral types $\left(0.85 \mathcal{M}_{\odot}\right.$ and $\left.0.68 \mathcal{M}_{\odot}\right)$. These results would corroborate the previous findings of Arenou et al. (2000), $0.83 \pm 0.09 \mathcal{M}_{\odot}$ and $0.79 \pm 0.09 \mathcal{M}_{\odot}$, respectively. Moreover, dynamical parallax (57.21 \pm 0.99 mas) in is good agreement with that derived by Söderhjelm (1999) from the intermediate transit data of HIPPARcos (57.6 \pm 0.6 mas).

19598-0957 (HO 276). This pair is a LWSB with spectral type F9 V (Gray et al. 2003). Individual masses obtained in the present work $\left(1.105 \pm 0.009 \mathcal{M}_{\odot}\right.$ and $\left.0.832 \pm 0.020 \mathcal{M}_{\odot}\right)$ are in reasonable agreement with those derived by Tokovinin (2017), $1.18 \mathcal{M}_{\odot}$ and $0.91 \mathcal{M}_{\odot}$, as well as with those estimated considering its spectral type along with a difference of magnitudes $\Delta m=1.606 \pm 0.133$ (about $1.2 \mathcal{M}_{\odot}$ and $0.9 \mathcal{M}_{\odot}$ ).

Dynamical parallax $(42.29 \pm 0.45 \mathrm{mas})$ is slightly closer to the orbital parallax $(40.21 \pm 5.74$ mas $)$ than to trigonometric measurements of both HIP2 (45.04 \pm 0.99 mas) and GDR2 $(46.9328 \pm 0.9290$ mas). This would be another case of biased trigonometric parallax likely due to the uncorrected orbital motion.

$21145+1000$ (STT 535 AB). The $\delta$ Equ is a F7 V + G0 V system according to Beavers \& Cook (1980) with a difference of magnitudes given by HIPPARCos, $\Delta m=0.331 \pm 0.232$. However, ten Brummelaar et al. (2000) determined $\Delta m=0.09 \pm 0.04$ using adaptive optics observations. Moreover, in accordance with their measurements, this system would be a F6 V + F6 V binary with a total mass of $2.35 \pm 0.12 \mathcal{M}_{\odot}$.

Several authors have estimated individual masses, as well as parallax, in agreement with these values. For example, Söderhjelm (1999) provided $1.21 \pm 0.07 \mathcal{M}_{\odot}$ and $1.13 \pm 0.06$ $\mathcal{M}_{\odot}$, respectively, along with a parallax of $54.32 \pm 0.90$ mas. Similar estimations were derived by Arenou et al. (2000) and Pourbaix (2000), the latter including a parallax of $55.00 \pm$ 0.67 mas. In addition, masses of $1.192 \pm 0.012 \mathcal{M}_{\odot}$ and $1.187 \pm$ $0.012 \mathcal{M}_{\odot}$, along with a parallax of $54.41 \pm 0.14$ mas, were obtained by Muterspaugh et al. (2008).

All of them are in complete agreement with those calculated in this work. We considered the above-mentioned $\Delta m$ given by ten Brummelaar et al. (2000) instead of that given by HipPARCos. As a result, we obtained individual masses of $1.214 \pm 0.008 \mathcal{M}_{\odot}$ and $1.193 \pm 0.012 \mathcal{M}_{\odot}$, respectively, along with a precise dynamical parallax of $54.21 \pm 0.12$ mas.

\section{Summary and conclusions}

Dynamical parallaxes are often the only way to obtain reliable individual masses and distances of binary systems. In this paper, we describe an exact theory to compute dynamical parallaxes and individual masses of visual binaries which takes the actual location of each component on the MS in the HR diagram into consideration. This was accomplished by defining a new nonlinear MLR by means of a polynomial of degree 20 which yields masses with a trueness of $10^{-11} \mathcal{M}_{\odot}$ and a precision of $0.014 \mathcal{M}_{\odot}$, covering the spectral range from O7 V to M9.5 V. For the sake of completeness, we give a new linear MLR as well.

The resulting model (ANAPAR) was validated using a synthetic sample comprising $10^{3}$ systems. The results offer overwhelming evidence for the superiority of this model with respect to the previous ones in terms of the accuracy in both parallaxes and individual masses.

We used this to obtain the most accurate dynamical parallaxes and masses for the $19 \mathrm{VB}+\mathrm{SB} 2$ with definitive orbits in the ORB6. Our findings appear to suggest that these values could be even more reliable than trigonometric measurements, particularly when trigonometric parallax measurements might be affected because their mutual orbital motions have not been taken into account.

Future work will involve the extension of the proposed model to binary systems with multiple subcomponents and with evolved companions.

Acknowledgements. I am grateful to A. Tokovinin for his helpful revision and comments on the manuscript. This paper was supported by the Spanish Ministerio de Economía, Industria y Competitividad under the Project AYA201680938-P (AEI/FEDER, EU). This research used the Washington Double Star Catalog maintained at USNO, the SIMBAD Astronomical Database operated by the Centre des Données Stellaires (Strasbourg, France), and bibliographic references from the Astrophysics Data System maintained by SAO/NASA. This work has made use of data from the European Space Agency (ESA) mission Gaia (https://www.cosmos.esa.int/gaia), processed by the Gaia Data Processing and Analysis Consortium (DPAC, https://www.cosmos.esa.int/ web/gaia/dpac/consortium). Funding for the DPAC has been provided by national institutions, in particular the institutions participating in the Gaia Multilateral Agreement.

\section{References}

Abt, H. A. 2009, ApJS, 180, 117

Agati, J.-L., Bonneau, D., Jorissen, A., et al. 2015, A\&A, 574, A6

Akaike, H. 1971, in Information Theory and an Extension of the Maximum

Likelihood Principle, 2nd Int. Symp. Inform. Theory, Tsahkadsor, 1971, 267 Andrade, M. 2007, Rev. Mex. Astron. Astrofis., 43, 237

Angelov, T. 1993, Bull. Astron. Belgrad., 148, 1

Arenou, F., Halbwachs, J.-L., Mayor, M., Palasi, J., \& Udry, S. 2000, IAU Symp., 200, 135

Baize, P., \& Romani, L. 1946, Ann. Astrophys., 9, 13

Baron, F., Monnier, J. D., Pedretti, E., et al. 2012, ApJ, 752, 20

Beavers, W. I., \& Cook, D. B. 1980, ApJS, 44, 489

Bidelman, W. P. 1985, ApJS, 59, 197

Brent, R. P. 1971, Comput. J., 14, 422

Burnham, K., \& Anderson, D. 2002, Model Selection and Multimodel Inference: A Practical Information-theoretic Approach (Berlin: Springer Verlag)

Couteau, P. 1978, L'observation des étoiles doubles visuelles, suivie d'un catalogue de 744 étoiles doubles pour tous instruments (Paris: Flammarion)

Dekker, T. J. 1969, in Constructive Aspects of the Fundamental Theorem of Algebra, in Proceedings of a Symposium Conducted at the IBM Research Laboratory, Zürich-Rüschlikon, Switzerland, June 5-7, 1967, eds. P. Henrici, \& B. Dejon (London: Wiley-Interscience)

Docobo, J. A., \& Andrade, M. 2006, ApJ, 652, 681

Docobo, J. A., \& Andrade, M. 2013, MNRAS, 428, 321

Docobo, J. A., \& Andrade, M. 2015, AJ, 149, 45

Duquennoy, A., \& Mayor, M. 1991, A\&A, 248, 485

Eggenberger, P., Miglio, A., Carrier, F., Fernandes, J., \& Santos, N. C. 2008, A\&A, 482, 631

ESA 1997, in The Hipparcos and Tycho Catalogues. Astrometric and Photometric Star Catalogues Derived from the ESA Hipparcos Space Astrometry Mission, ESA SP, 1200

Farrington, C. D., ten Brummelaar, T. A., Mason, B. D., et al. 2010, AJ, 139, 2308

Farrington, C. D., ten Brummelaar, T. A., Mason, B. D., et al. 2014, AJ, 148, 48 Gaia Collaboration (Brown, A. G. A., et al.) 2016, A\&A, 595, A2 Gaia Collaboration (Brown, A. G. A., et al.) 2018, A\&A, 616, A1 Geyer, D. W., Harrington, R. S., \& Worley, C. E. 1988, AJ, 95, 1841 Gray, R. O., Napier, M. G., \& Winkler, L. I. 2001, AJ, 121, 2148 
M. Andrade: Dynamical parallaxes and individual masses of main-sequence visual binaries

Gray, R. O., Corbally, C. J., Garrison, R. F., McFadden, M. T., \& Robinson, P. E 2003, AJ, 126, 2048

Gray, R. O., Corbally, C. J., Garrison, R. F., et al. 2006, AJ, 132, 161

Griffin, R. F. 2012, JApA, 33, 29

Halbwachs, J.-L., Mayor, M., \& Udry, S. 2018, A\&A, 619, A81

Harlan, E. A. 1974, AJ, 79, 682

Hartkopf, W. I., \& Mason, B. D. 2010, IAU Commission on Double Stars, 170, 1

Hartkopf, W. I., Mason, B. D., \& Worley, C. E. 2001, AJ, 122, 3472

Heintz, W. D. 1962, Veroeffentlichungen der Sternwarte Munchen, 5, 135

Heintz, W. D. 1971, Publ. Leander McCormick Obs., 16, 283

Heintz, W. D. 1987, AJ, 94, 1077

Heintz, W. D. 1994, AJ, 108, 2338

Hill, G., Barnes, J. V., Hutchings, J. B., \& Pearce, J. A. 1971, ApJ, 168, 443

Houk, N., \& Smith-Moore, M. 1988, Michigan Catalogue of Two-dimensional Spectral Types for the HD Stars. Volume 4, Declinations $-26^{\circ} 0$ to -12.0

JCGM:101. 2008, Evaluation of Measurement Data-Supplement 1 to the "Guide to the Expression of Uncertainty in Measurement" - Propagation of Distributions using a Monte Carlo Method, Tech. rep. (JCGM)

Joy, A. H., \& Abt, H. A. 1974, ApJS, 28, 1

Kacker, R. N., Kessel, R., \& Sommer, K.-D. 2010, J. Res. Natl. Inst. Stand Technol., 115, 453

Keenan, P. C., \& McNeil, R. C. 1989, ApJS, 71, 245

Kervella, P., Mérand, A., Ledoux, C., Demory, B. O., \& Le Bouquin, J. B. 2016, A\&A, 593, A127

Kiefer, F., Halbwachs, J.-L., Lebreton, Y., et al. 2018, MNRAS, 474, 731

Martin, C., \& Mignard, F. 1998, A\&A, 330, 585

Mason, B. D., \& Hartkopf, W. I. 2005, IAU Commission on Double Stars, 156 1

Mason, B. D., \& Hartkopf, W. I. 2012, IAU Commission on Double Stars, 178, 1
Mazeh, T., Latham, D. W., Goldberg, E., et al. 2001, MNRAS, 325, 343 McCluskey, Jr., G. E., \& Kondo, Y. 1972, Ap\&SS, 17, 134

Muterspaugh, M. W., Lane, B. F., Fekel, F. C., et al. 2008, AJ, 135, 766 Muterspaugh, M. W., Hartkopf, W. I., Lane, B. F., et al. 2010, AJ, 140, 1623

Parenago, P. P. 1945, Pop. Astron., 53, 441

Pecaut, M. J., \& Mamajek, E. E. 2013, ApJS, 208, 9

Pourbaix, D. 2000, A\&AS, 145, 215

Pourbaix, D., Tokovinin, A. A., Batten, A. H., et al. 2004, A\&A, 424, 727

Richards, M. T. 1993, ApJS, 86, 255

Russell, H. N., \& Moore, C. E. 1940, The Masses of the Stars (Chicago: University of Chicago Press)

Ségransan, D., Delfosse, X., Forveille, T., et al. 2000, A\&A, 364, 665

Sharov, A. S. 1964, Sov. Astron., 7, 689

Söderhjelm, S. 1999, A\&A, 341, 121

Strassmeier, K. G., Hall, D. S., Fekel, F. C., \& Scheck, M. 1993, A\&AS, 100, 173

Struve, O., \& Franklin, K. L. 1955, ApJ, 121, 337

Sugiura, N. 1978, Methods, 7, 13

ten Brummelaar, T., Mason, B. D., McAlister, H. A., et al. 2000, AJ, 119, 2403

Tokovinin, A. 2012, AJ, 144, 56

Tokovinin, A. 2016, AJ, 152, 10

Tokovinin, A. 2017, AJ, 154, 110

Tokovinin, A. A., Griffin, R. F., Balega, Y. Y., Pluzhnik, E. A., \& Udry, S. 2000, Astron. Lett., 26, 116

Torres, G. 2010, AJ, 140, 1158

van Altena, W. F., Lee, J. T., \& Hoffleit, E. D. 1995, The General Catalogue of Trigonometric Stellar Parallaxes (New Haven, CT: Yale University Observatory)

van Leeuwen, F. 2007, A\&A, 474, 653

Zavala, R. T., Hummel, C. A., Boboltz, D. A., et al. 2010, ApJ, 715, L44 